\title{
ON THE MAGNITUDE OF FOURIER COEFFICIENTS
}

\author{
MICHAEL SCHRAMM AND DANIEL WATERMAN
}

\begin{abstract}
If $f$ is a function on $R^{1}$ of $\Lambda$-bounded variation and period $2 \pi$, then its $n$th Fourier coefficient $\hat{f}(n)=O\left(1 / \Sigma_{1}^{n} 1 / \lambda_{j}\right)$ and its integral modulus of continuity $\omega_{1}(f ; \delta)=O\left(1 / \Sigma_{1}^{[1 / \delta]} 1 / \lambda_{j}\right)$. The result on $\hat{f}(n)$ is best possible in a sense. These results can be extended to certain other classes of functions of generalized variation.
\end{abstract}

For various classes of functions, estimates of the magnitude of the Fourier coefficients and of the integral moduli of continuity can be made. Here we shall do this for certain classes of functions of generalized bounded variation.

1. Let us suppose that $f$ is a real-valued function on an interval $I$ of $R^{1}$. If $I_{n}=\left[a_{n}, b_{n}\right] \subset I$, set $f\left(I_{n}\right)=f\left(b_{n}\right)-f\left(a_{n}\right)$. We suppose the intervals $I_{n}, n=$ $1,2, \ldots$, to be nonoverlapping. If $\Lambda=\left\{\lambda_{n}\right\}$ is a nondecreasing sequence of positive real numbers such that $\sum 1 / \lambda_{n}=\infty$, we have said [5] that $f$ is of $\Lambda$-bounded variation $(\Lambda \mathrm{BV})$ if, for every $\left\{I_{n}\right\}$,

$$
\sum\left|f\left(I_{n}\right)\right| / \lambda_{n}<\infty \text {. }
$$

If $f \in \Lambda \mathrm{BV}$ on $[a, b]$ it is known [5] that the supremum of the sums (1) is finite. This supremum is called the $\Lambda$-variation of $f$ on $[a, b], V_{\Lambda}(f ; a, b)$.

If $\phi$ is a nonnegative convex function defined on $[0, \infty)$ such that $\phi(x) / x \rightarrow 0$ as $x \rightarrow 0$, we shall say that $f$ is of $\phi \Lambda$-bounded variation $(\phi \Lambda \mathrm{BV})$ if there is a $c>0$ such that, for every $\left\{I_{n}\right\}$,

$$
\sum \phi\left(c\left|f\left(I_{n}\right)\right|\right) / \lambda_{n}<\infty
$$

For $\phi(x)=x^{p}, p>1$, this class has been studied by Shiba [2], who calls it $\Lambda \mathrm{BV}^{(p)}$.

For $p \geq 1$, we define the integral modulus of continuity of order $p$ to be

$$
\omega_{p}(f ; \delta)=\sup _{0<t \leq \delta}\left(\int_{I}|f(x+t)-f(x)|^{p} d x\right)^{1 / p} .
$$

We have previously estimated the Fourier coefficients of functions in $\Lambda \mathrm{BV}$. For $\Lambda=\left\{n^{1+\beta}\right\},-1<\beta<0$, we showed that $\hat{f}(n)=O\left(n^{\beta}\right)$ by showing that the Fourier series of such $f$ are $(C, \beta)$-bounded [4]. We have shown in general [6] that

$$
\hat{f}(n)=O\left(\lambda_{n} / n\right),
$$

which in the cases $\lambda_{n} \equiv 1$ (ordinary bounded variation) and $\lambda_{n}=n^{1+\beta}$ agrees with previous estimates. It is clear that in some cases (3) is not an adequate estimate. For example, in the case $\lambda_{n}=n$, the functions of harmonic bounded variation (HBV),

Received by the editors March 22, 1981. Presented to the Society, January 16, 1982.

1980 Mathematics Subject Classification. Primary 42A16; Secondary 26A45. 
it yields no useful information. Here we show $\hat{f}(n)=O\left(1 / \sum_{1}^{n} 1 / \lambda_{j}\right)$ which, in the case of $\mathrm{HBV}$, yields $\hat{f}(n)=O(1 / \log n)$.

This estimate of the Fourier coefficients of functions of $\Lambda \mathrm{BV}$ is best possible, in a sense made clear by Theorem 2 .

We now state our results.

THEOREM 1. Let $f: R^{1} \rightarrow R^{1}$ be of period $2 \pi$;

(i) if $f \in \Lambda \mathrm{BV}$, then $\omega_{1}(f ; \delta)=O\left(1 / \sum_{1}^{[1 / \delta]} 1 / \lambda_{j}\right)$;

(ii) if $f \in \phi \Lambda \mathrm{BV}$, then $\omega_{1}(f ; \delta)=O\left(\phi^{-1}\left(1 / \sum_{1}^{[1 / \delta]} 1 / \lambda_{j}\right)\right)$;

(iii) if $f \in \Lambda \mathrm{BV}^{(p)}, 1 \leq p<\infty$, then $\omega_{p}(f ; \delta)=O\left(1 /\left(\sum_{1}^{[1 / \delta]} 1 / \lambda_{j}\right)^{1 / p}\right)$.

Clearly,

$$
\begin{aligned}
\int_{0}^{2 \pi} f(x) e^{-i n x} d x & =-\int_{0}^{2 \pi} f\left(x+\frac{\pi}{n}\right) e^{-i n x} d x \\
& =\frac{1}{2} \int_{0}^{2 \pi}\left(f(x)-f\left(x+\frac{\pi}{n}\right)\right) e^{-i n x} d x
\end{aligned}
$$

and

$$
|\hat{f}(n)| \leq \frac{1}{4 \pi} \int_{0}^{2 \pi}\left|f(x)-f\left(x+\frac{\pi}{n}\right)\right| d x \leq \frac{1}{4 \pi} \omega_{1}\left(f ; \frac{\pi}{n}\right) .
$$

Since $\sum_{1}^{n} 1 / \lambda_{j} \leq 6 \sum_{1}^{[n / \pi]} 1 / \lambda_{j}$ for $n \geq 4$, we see that Theorem 1 has the following

COROLlaRY. If $f \in \Lambda \mathrm{BV}$, then $\hat{f}(n)=O\left(1 / \sum_{1}^{n} 1 / \lambda_{j}\right)$; if $f \in \phi \Lambda \mathrm{BV}$, then $\hat{f}(n)=O\left(\phi^{-1}\left(1 / \sum_{1}^{n} 1 / \lambda_{j}\right)\right)$.

The following expresses the sense in which this estimate is best possible.

THEOREM 2. If $\Gamma \mathrm{BV} \nsupseteq \Lambda \mathrm{BV}$, then there is an $f \in \Gamma \mathrm{BV}$ such that $\hat{f}(n) \neq$ $O\left(1 / \sum_{1}^{n} 1 / \lambda_{j}\right)$.

We have recently learned that these results, insofar as they apply to $\Lambda \mathrm{BV}$, have also been obtained by Wang Si-lei [3].

2. We turn now to the proof of Theorem 1 .

For any $f \in \Lambda \mathrm{BV}$, setting $k=[1 / t]$ for $0<t \leq \delta$, we observe

$$
\begin{aligned}
\int_{0}^{2 \pi} \mid f(x) & -f(x+t)\left|d x=\int_{0}^{2 \pi}\right| f(x+(j-1) t)-f(x+j t) \mid d x \\
& =\left(1 / \sum_{1}^{k} 1 / \lambda_{j}\right) \int_{0}^{2 \pi}\left(\sum_{j=1}^{k}|f(x+(j-1) t)-f(x+j t)| / \lambda_{j}\right) d x \\
& \leq 2 \pi V_{\Lambda}(f ; 0,4 \pi) / \sum_{1}^{k} 1 / \lambda_{j} \\
& \leq 2 \pi V_{\Lambda}(f ; 0,4 \pi) / \sum_{1}^{[1 / \delta]} 1 / \lambda_{j} .
\end{aligned}
$$

The first part of Theorem 1 is then established. 
If $f \in \phi \Lambda \mathrm{BV}$, then, for $c>0$ and sufficiently small

$$
\begin{aligned}
& \phi\left(\frac{c}{2 \pi} \int_{0}^{2 \pi}|f(x)-f(x+t)| d x\right) \leq \frac{1}{2 \pi} \int_{0}^{2 \pi} \phi(c|f(x)-f(x+t)|) d x \\
& \quad=\left(1 / \sum_{1}^{k} 1 / \lambda_{j}\right)\left(\frac{1}{2 \pi} \int_{0}^{2 \pi}\left(\sum_{j=1}^{k} \phi(c|f(x+(j-1) t)-f(x+j t)|) / \lambda_{j}\right) d x\right) \\
& \quad \leq V_{\phi \Lambda}(c f ; 0,4 \pi) / \sum_{1}^{k} 1 / \lambda_{j}
\end{aligned}
$$

where the numerator denotes the supremum of sums of the form (2). Since $\phi$ is convex, $\phi(\alpha x)<\alpha \phi(x)$ for $0<\alpha<1$. Thus we may choose $c$ so that $V_{\phi \Lambda}(c f) \leq 1$; then

$$
\int_{0}^{2 \pi}|f(x)-f(x+t)| d x \leq \frac{2 \pi}{c} \phi^{-1}\left(1 / \sum_{1}^{k} 1 / \lambda_{j}\right) \leq \frac{2 \pi}{c} \phi^{-1}\left(1 / \sum_{1}^{[1 / \delta]} 1 / \lambda_{j}\right),
$$

and the second part of Theorem 1 has been established. For $f \in \Lambda \mathrm{BV}^{(p)}$,

$$
\begin{aligned}
\int_{0}^{2 \pi} \mid f(x) & -\left.f(x+t)\right|^{p} d x \\
& =\left(1 / \sum_{1}^{k} 1 / \lambda_{j}\right) \int_{0}^{2 \pi}\left(\sum_{j=1}^{k}|f(x+(j-1) t)-f(x+j t)|^{p} / \lambda_{j}\right) d x
\end{aligned}
$$

and the argument is concluded just as above.

The corollary follows from the remarks which precede it and Theorem 1.

3. We now prove Theorem 2.

From a result of Perlman and Waterman [1], we know that $\Gamma \mathrm{BV} \nsupseteq \Lambda \mathrm{BV}, \Gamma=$ $\left\{\gamma_{n}\right\}$, implies $\sum_{1}^{n} 1 / \lambda_{j} \neq O\left(\sum_{1}^{n} 1 / \gamma_{j}\right)$.

Let $T_{n}(f)=\left(\sum_{1}^{n} 1 / \lambda_{j}\right) \int_{0}^{2 \pi} f(x) \sin n x d x$. We will show that there is an $f \in$ $\Gamma \mathrm{BV}$ such that $T_{n}(f) \neq O(1)$.

We define $f_{n}$ on $[0,2 \pi)$ by

$$
f_{n}(x)=(-1)^{k+1} / 4 \sum_{j=1}^{n} 1 / \gamma_{j} ; \quad(k-1) \pi / n \leq x<k \pi / n, k=1,2, \ldots, 2 n,
$$

and extend $f_{n}$ to $R^{1}$ with period $2 \pi$. Then

$$
V_{\Gamma}\left(f_{n} ; 0,2 \pi\right)=\left(\sum_{1}^{2 n} 1 / \gamma_{j}\right) /\left(2 \sum_{1}^{n} 1 / \gamma_{j}\right) \leq 1,
$$

while

$$
\begin{aligned}
T_{n}\left(f_{n}\right) & =\left(\sum_{1}^{n} 1 / \lambda_{j}\right)\left(1 / 4 \sum_{1}^{n} 1 / \gamma_{j}\right) \int_{0}^{2 \pi}|\sin n x| d x \\
& =\left(\sum_{1}^{n} 1 / \lambda_{j}\right) /\left(\sum_{1}^{n} 1 / \gamma_{j}\right) \neq O(1),
\end{aligned}
$$

and the result follows from the Banach-Steinhaus Theorem. 


\section{REFERENCES}

1. S. Perlman and D. Waterman, Some remarks on functions of $\Lambda$-bounded variation, Proc. Amer. Math. Soc. 74 (1979), 113-118.

2. M. Shiba, On absolute convergence of Fourier series of functions of class $\Lambda \mathrm{BV}^{(p)}$, Sci. Rep. Fukushima Univ. 30 (1980), 7-10.

3. S. Wang, Personal communication.

4. D. Waterman, On the summability of Fourier series of functions of $\Lambda$-bounded variation, Studia Math. 55 (1976), 87-95.

5. __, On $\Lambda$-bounded variation, Studia Math. 57 (1976), 33-45.

6. $\ldots$, Fourier series of functions of $\Lambda$-bounded variation, Proc. Amer. Math. Soc. 74 (1979), 119-123.

Department of Mathematics, Syracuse UNIVERsity, Syracuse, New York 13210 